\title{
Iris Recognition System with Accurate Eyelash Segmentation \& Improved FAR, FRR using Textural \& Topological Features
}

\author{
Archana V Mire \\ Asst Prof dept of IT ,Bapurao \\ Deshmukh College of Engineering, \\ Sevagram \\ Nagpur University, Maharashtra, India
}

\author{
Bharti L Dhote \\ Asst Prof Sinhgad Institute of \\ Technology, Lonavala \\ Pune University, Maharashtra, India
}

\begin{abstract}
This paper represents novel iris recognition technique which uses textural and topological features. Converting circular iris pattern into rectangular pattern makes it rotation invariant. Most of the research in iris recognition is on encoding and recognition of iris pattern but segmenting exact iris pattern is itself very tedious task in this paper we are trying to emphasize on better iris segmentation technique. In other systems performance of the system is always dependent on threshold. There is always conflict between FAR \& FRR, if tied to improve one quantity degrades other one. This paper describes an alternate means to identify individuals using images of their iris with low false acceptance rate and low false rejection rate. For encoding topological feature Euler vector can be utilized while for encoding textural feature histogram is used. Histogram is matched by using Du measure whose origin belong in Hyperspectral Image Analysis while for matching euler vector Vector Difference Matching algorithm is developed .
\end{abstract}

\section{Keywords}

Histogram, Du measure, Euler vector, FAR, FRR

\section{INTRODUCTION}

Iris texture patterns are believed to be different for each person, and even for the two eyes of the same person. It is also claimed that for a given person, the iris patterns change little after youth. Very high recognition/verification rates have been reported for iris recognition systems in studies to date. For Daugman's system, when choosing the Hamming distance (HD) matching threshold value of 0.32 , the false accept rate (FAR) was decreased from 1 in 151,000 (1993) to 1 in 26 million (2003) [1] [2]. On the basis of these conceptual claims and empirical reports, iris is often thought to be one of the highest-accuracy biometrics. Compared with some other biometrics, such as fingerprints and face, iris recognition has a relatively short history of use. The present day iris recognition systems offer reliable and effective security but these systems are low on user confidence, user friendliness and have a high False Rejection Rate (FRR). One constraint of current iris recognition systems, which is perhaps not widely appreciated, is that they require substantial user cooperation in order to acquire an image of sufficient quality for use. In this paper we present a novel iris verification algorithm which uses textural and topological features of the iris image. Histogram is used to extract the textural information [2] and Euler numbers are used to extract the topological information from the iris image [3] . One constraint of current iris recognition systems, which is perhaps not widely appreciated, is that they require substantial user cooperation in order to acquire an image of sufficient quality for use. We have Du measure and Euler vector to match the textural and topological information. Based on these algorithms, a matching strategy is presented to reduce the false rejection while false acceptance is unaffected. The algorithm is tested on the CASIA iris image database [4].

\section{IRIS SEGMENTATION}

For most algorithms, and assuming near-frontal presentation of the pupil, the iris boundaries are modeled as two circles, which are not necessarily concentric. The inner circle is the boundary between the pupil and the iris. The outer circle is the boundary between the iris and the sclera. To avoid eyebrows while locating pupil and iris first eye image is rotated with 180 degrees.

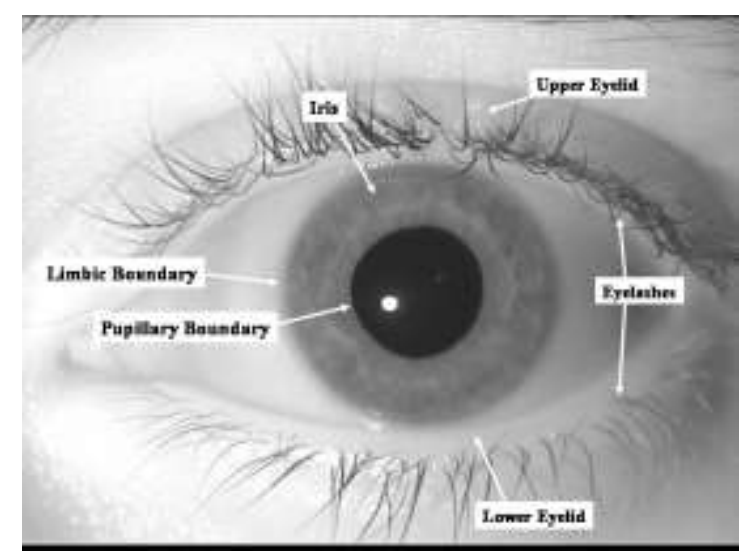

Figure 1 Introduction with Iris

\section{Locating Iris inner boundary}

Iris inner boundary is nothing but pupil circumference so if we can get centre \& radius of pupil it is as good as locating iris inner boundary. All the pixels in the image having intensity greater than 70 are marked as 1(255) while pixels having intensity less than 70 are marked as 0 . Resultant image consist of dark pupil along with some dark pigments on screen (which may be due to eyelid \& eyelash). Image is negated so that pupil will be bright and all other pixels will be negative. From the image connected component are marked and component having maximum no of connections is marked as pupil. Centroid of connected component gives the center of pupil. Once the centre of pupil known, pixels along horizontal and vertical direction is checked to find pupil radius, maximum of the two is marked as final radius of the pupil. 


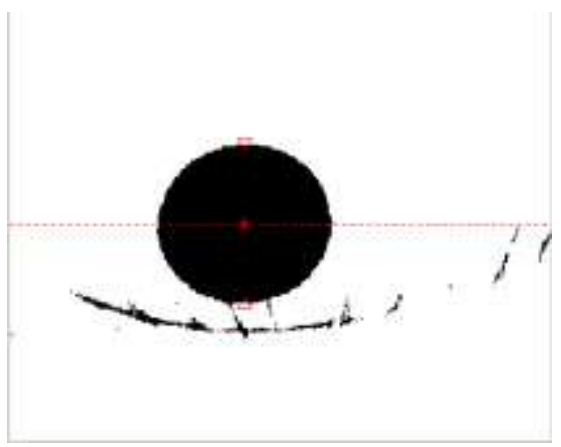

Figure 2 Segmented Pupil, centre \& radius

\subsection{Locating Iris Outer boundary}

For locating Iris boundary Canny edge detection is performed on the image again followed by gamma adjustment, non maximum suppression and hysteresis thresholding . On the resultant image Houghs transform for circle detection is performed which gives nothing but iris outer boundary.
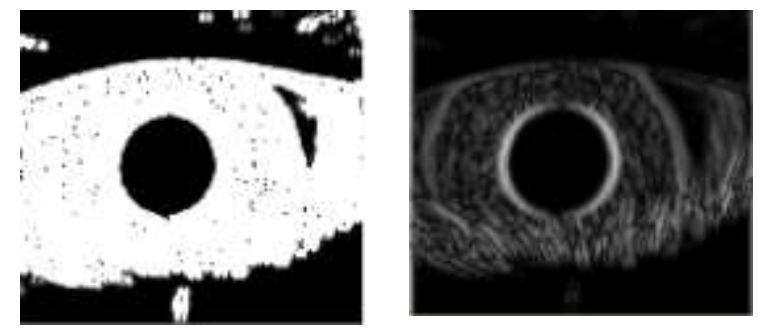

a)Canny Edge detection

b)Gamma adjustment
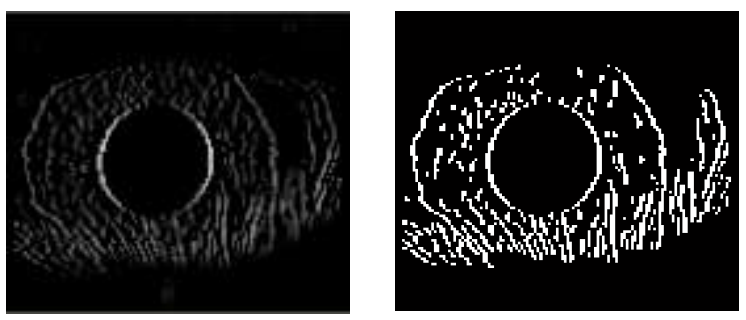

c)Non maximum

d)Hysterysis thesholding

Figure 3 processing iris for Iris Edge detection

On the resultant image Houghs transform for circle detection is performed which gives nothing but iris outer boundary.

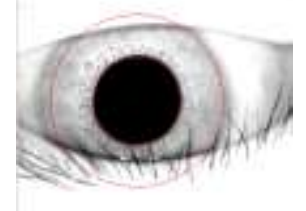

a)Iris outer boundary located using houghs transform

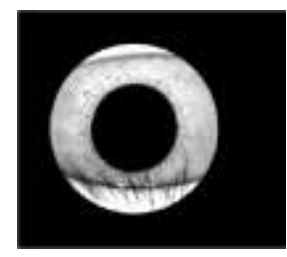

b)Segmented iris

Figure 4 Iris Segmentation

\section{EYELASH SEGMENTATION}

Two classes of eyelashes are defined in our eyelash detection model, separable and multiple eyelashes. Separable eyelashes are defined as the eyelashes that can be distinguished from other eyelashes and multiple eyelashes are the eyelashes that overlap in a small area. Fig. 5 illustrates the two classes of eyelashes.

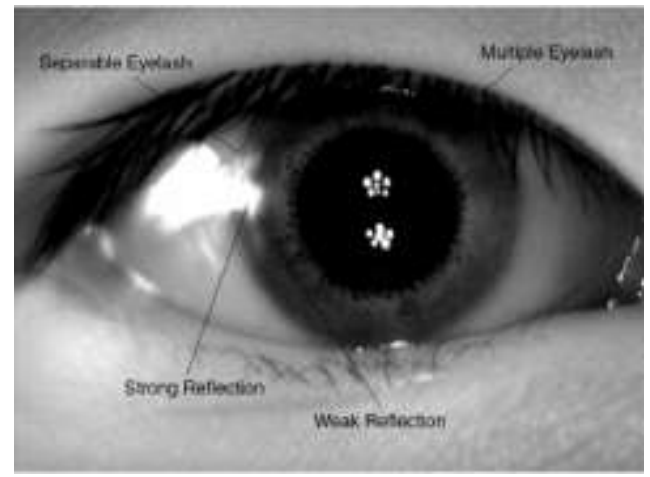

Figure 5 multiple \& separable Eyelashes

\subsection{Separable Eyelash}

By the definition of separable eyelashes, they can be distinguished from other eyelashes; thus, the pixels around separable eyelash should not belong to other eyelashes. In fact, most of pixels around separable eyelashes are iris pixels. Because of the intensity difference between iris pixels and eyelashes pixels, a separable eyelash can be regarded as an edge in an image. Fig. 5 shows a cross section of a separable eyelash gray level and part of iris around it. Based on this property, a real part of Gabor filter is proposed to detect separable eyelashes, which, in the spatial domain has the following general form,

$$
G(x, u, \sigma)=\exp x^{2} / 2 \sigma^{2} \cos (2 \pi u x)
$$

Where $\mathrm{u}$ is the frequency of the sinusoidal wave and $\sigma$ is the standard derivation of the Gaussian envelope. The resultant values are small when a separable eyelash convolutes with the filter. In fact, the filter serves as an edge detector. If a resultant value of a point is smaller than a threshold, it is noted that this point belongs to an eyelash. Mathematically, it can be represented by

$$
F(x)=f(x) * G(v, u, s)<K_{l}
$$

\section{Eq2}

Where $\mathrm{K}_{1}$ is a pre-defined threshold that is -45 using experiments and $*$ represents an operator of convolution.

\subsection{Multiple Eyelashes}

For multiple eyelashes, many eyelashes overlap in a small area, which results in less intensity variation in this area. Thus, for detecting multiple eyelashes, if the variance of intensity in the area is less than a threshold, the center of the window is noted as a pixel of eyelash. It can be described by,

$\frac{\sum_{i=-N}^{N} \sum_{j=-N}^{N}(f(x+i, y+j)-M)^{2}}{(2 N+1)^{2}}<\mathrm{K}_{2}$ 
Where $\mathrm{M}$ is the mean of intensity in the small window; $(2 \mathrm{~N}+1)^{2}$ is the window size and $\mathrm{K}_{2}$ is a threshold. In the following experiments, $\mathrm{K}_{2}$ is defined as 6 and $(2 \mathrm{~N}+1)^{2}$ as $5 \times 5$.

\subsection{Connective Property}

In order to provide more robust and accurate detection method, the connective property of an eyelash is utilized to avoid misclassification from the previous inequalities. Each point in an eyelash should connect to another eyelash point or to an eyelid. If any point fulfills one of the two previous inequalities, its neighbor pixels are required to check whether they belong to an eyelash or eyelid. If none of the neighbor pixels has been classified as a point in an eyelid or an eyelash,

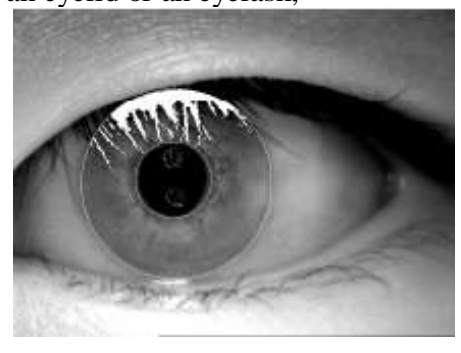

a)Eyelash detected

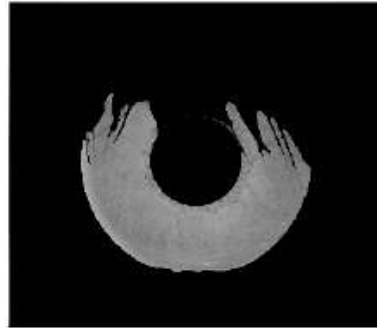

b)Segmented Iris with removed eyelash
Figure 6 Eyelash segmentation

\section{IRIS IMAGE NORMALIZATION}

The image is converted to standard rectangle strips using the radial scan method[5], which is used to collect all pixels in the iris portion and produce trapezoidal shape image as shown in figure 7b. Equation 4 describes scanning process of radial scan module.

$$
\begin{aligned}
& x=\oint x_{c}+r_{i} \cos ((\pi / 180) \theta) \\
& y=\oint y_{c}+r_{i} \sin ((\pi / 180) \theta)
\end{aligned}
$$

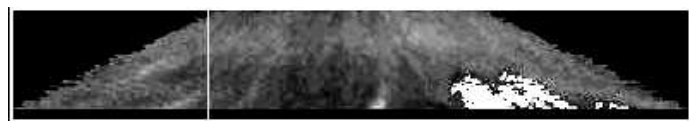

a)Process of radial scan method

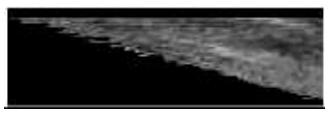

b)output of radial process

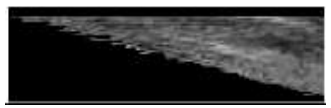

c)flopping operation for forming fixed size iris strip

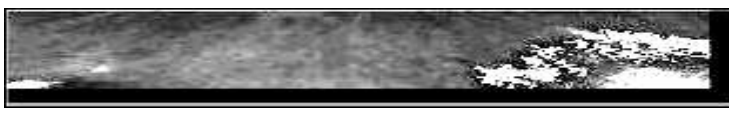

d)result of iris normalization process

\section{Figure 7 Converting circular iris pattern into rectangular form}

Where $\mathrm{x}$ and $\mathrm{y}$ are polar coordinates of the trapezoidal pattern, $\mathrm{x}_{\mathrm{c}}$ and $\mathrm{y}_{\mathrm{c}}$ are the centre of the circular iris pattern, $\mathrm{r}_{\mathrm{i}}$ is a radius of $\mathrm{i}^{\text {th }}$ scan. After forming trapezoidal pattern, the left triangular portion of the image is removed and mapped into the right portion of the iris image. After this process, dilation or erosion module can be performed to form a fixed size rectangle strip. Figure 7(ad) describes various steps involved in the iris normalization process

\section{IRIS ENCODING}

Two reduce false acceptance rate and false rejection rate two way encoding of iris is performed once by using histogram and other by using Euler number. Both histogram and Euler vector are orientation independent so this approach provides orientation independency to iris encoding and recognition. Steps for performing histogram analysis and Euler vector computation are as follows.

\subsection{Histogram Analysis}

The histogram of a segmented image (such as seen in Fig. 7), $\mathrm{H}_{0}[\mathrm{n}]$, is then computed. Since the segmented image contains primarily zero pixel values, and the pupil itself has very low values, the histogram is modified to remove the effects of these pixels. In addition, there may be reflections (very high pixel values) that were not removed in the preprocessing that should be accounted for. This modification is described as:

$$
\begin{gathered}
H_{1}=0 \\
H_{1}[n]=H_{0}[n] \\
H_{1}[n]=0
\end{gathered} \quad\left\{\begin{array}{c}
n<20 \\
20 \leq n \leq 230 \\
n>230
\end{array}\right.
$$

The resulting histogram for the iris segmented in Fig.7 is displayed in Fig. 8.

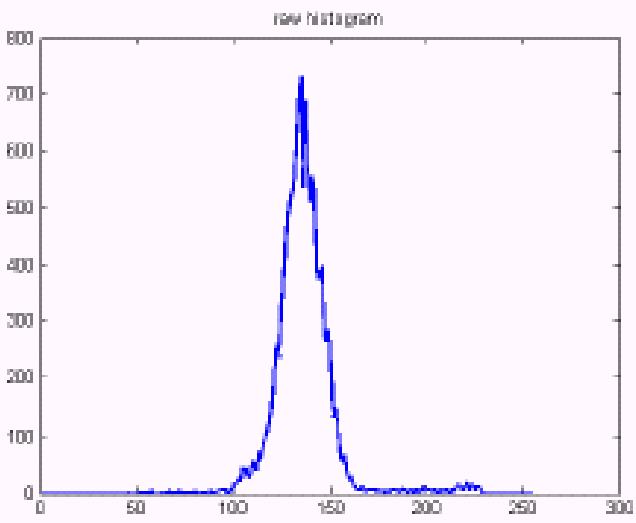

Figure 8 Histogram

To reduce noise, this result is then filtered with a 5-tap averaging filter. Further normalization is applied to each histogram. First, all histograms are scaled so that their peak value is 1.0

$$
\mathrm{H}_{2}[\mathrm{n}]=\mathrm{H}_{1}[\mathrm{n}] / \max \left\{\mathrm{H}_{1}[\mathrm{n}]\right\} \quad \mathrm{Eq} 6
$$


Finally, to adjust for illumination differences between images, the peak is shifted to occur at a grayscale value of 128 . This ensures that any two images of the same iris taken in some manner in order to produce a representative sample that has less noise than any individual image. For this system, the normalized histograms of three iris images are averaged, and the result stored in the database as the template for which comparisons are made for identification. An example of three normalized histograms of the same iris and the resulting template is shown in Fig.9

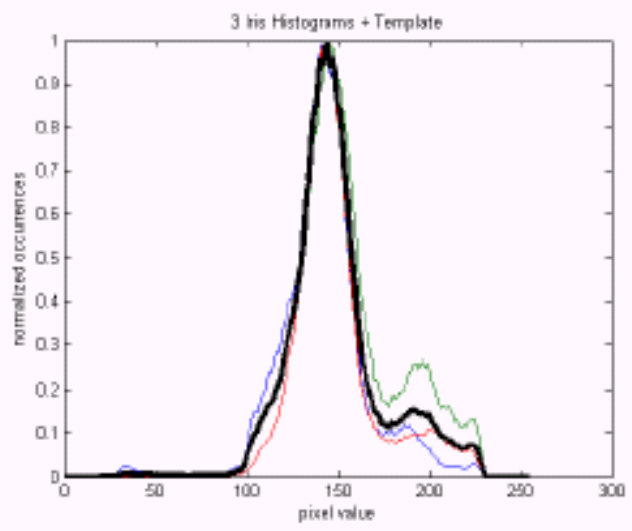

Figure 9 Normalized Histogram

\subsection{Euler vector computation}

For calculating Euler number gray levels of an $\mathrm{m}$ bit gray scale image can be represented in the form of base 2 polynomial[3]

$\mathrm{a}_{\mathrm{m}-1} 2^{\mathrm{m}-1}+\mathrm{a}_{\mathrm{m}-2} 2^{\mathrm{m}-2}+\ldots \ldots \mathrm{a}_{1} 2^{1}+\mathrm{a}_{0} 2^{0}$. Based on this property normalized iris image is decomposed into a collection of binary m 1-bit biplanes. For comparing two polar iris images using Euler Code, a common mask is generated. This common mask is obtained by performing a bitwise-OR operation of the two individual masks and is applied to both the polar images. For generating the Euler Code, four binary images corresponding to the four Most Significant Bits (MSB) of the masked polar iris image are considered. As shown in Figure 10, binary images are obtained by considering the four MSBs of every pixel of masked polar iris image separately. The Euler number which represents the difference between the number of connected components and the number of holes is computed for these binary images. This extracts the topological property of the iris image and is useful for global description of regions in the image. The topological feature is unaffected by common transformation and rotation. Euler Code is a vector matrix consisting of Euler numbers calculated from the binary images. Table 1 shows the Euler Codes.

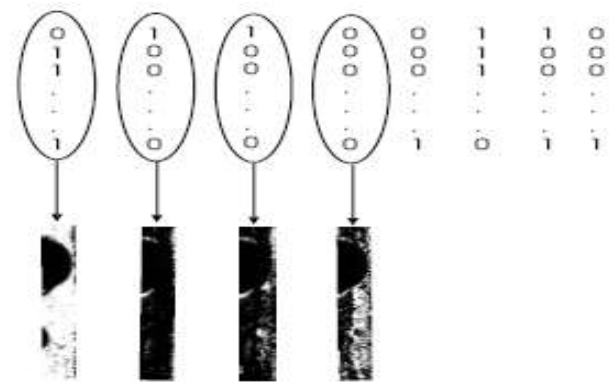

Figure 10 Biplanes for calculating Euler number
Table 1 Calculated Euler Vector

\begin{tabular}{|l|l|l|l|l|}
\hline \multicolumn{5}{|c|}{ EULER VECTOR } \\
\hline Image1 & -72 & 64 & 140 & -365 \\
\hline Image2 & -62 & 84 & 148 & -371 \\
\hline Image3 & -63 & 75 & 145 & -368 \\
\hline
\end{tabular}

\section{IRIS MATCHING}

As a new iris image is presented to the system, it undergoes the same preprocessing that went into each enrollment iris image. Histogram and Euler vectors are computed and matching is performed as below.

\subsection{Histogram Matching}

Its normalized histogram is then compared to each template in the enrollment database to determine if there is a match. The metric used to compare the two is the Du Measure, which has its origins in Hyperspectral/multispectral imaging [6][7][8]. The Du measure is defined as the product of the Spectral Angle Mapper (SAM), the Spectral Information Divergence (SID), and the average absolute difference between two vectors. The SAM between two vectors $r$ and $s$ is defined as

$$
\operatorname{SAM}(\vec{r}, \vec{s})=\cos ^{-1}\left[\begin{array}{ccc}
\langle\vec{r}, \vec{s}\rangle \\
\hline\|\vec{r}\| & \times & \|\vec{s}\|
\end{array}\right] \quad \text { Eq7 }
$$

While the SID between the same two vector is defined as

$$
\operatorname{SID}(\vec{r}, \vec{s})=D(\vec{p} \mid \vec{q})+D\langle\vec{p} \mid \vec{q}\rangle \quad \text { Eq8 }
$$

Where $D(q \| p)=\Sigma j=1\left(q_{j} / p_{j}\right)$ is a measure of entropy . The Du measure is defined as the product of the tangent of the SAM, the SID and the average absolute difference (AD) between the two vectors

$\operatorname{Du}(\mathrm{r}, \mathrm{s})=\operatorname{AD}(\mathrm{r}, \mathrm{s}) * \operatorname{SID}(\mathrm{r}, \mathrm{s}) * \tan (\operatorname{SAM}(\mathrm{r}, \mathrm{s})) \quad \mathrm{Eq} 9$

The result is a real number that represents the closeness of the two vectors. If the Du measure is relatively small, the two vectors are close. In this application, the two vectors are the two normalized histograms that are to be compared. Since the processing of the histograms includes zeroing the numbers of occurrences for grayscale values lower than 20 and values greater than 230, the two vectors that are compared each have 210 elements. If the Du value computed between a test iris histogram and a template in the database is less than the threshold, then the test iris was considered a match. A lower threshold will tend to reduce false acceptances but will also increase false rejections, and vice versa. Two reduce false acceptance rate threshold is kept at lower value $\alpha$ but whenever iris doesn't match threshold value is increased to $\beta$ and matching is performed once again if two(r,s ) matches with this threshold value matching can be performed once again by using Euler number .

\subsection{Matching using Euler number}

The Vector Difference Matching algorithm is designed to match two Euler Codes. In this algorithm, a comparison matrix is created with binary elements. This matrix stores the results of comparison of input Euler Code to the Euler Code from the database. For comparing Euler numbers the following equation is used:

$$
\left|\mathrm{Y}_{1}-\mathrm{Y}_{2} \leq \varepsilon\right|
$$

Where $\varepsilon$ is the tolerant error, $\mathrm{Y}_{1}$ and $\mathrm{Y}_{2}$ are the Euler numbers from the input Euler Code and the stored Euler Code respectively. 
The comparison matrix is initialized with all zeros. If the difference between $Y_{1}$ and $Y_{2}$ is less than the tolerant error $\varepsilon$ then one is entered in the comparison matrix. Table 2, Table 3 illustrates the procedure for generating the comparison matrix when the input is from a genuine user and an imposter (at $\varepsilon=20$ ).

Table 2

\begin{tabular}{|l|l|l|l|l|}
\hline & \multicolumn{4}{|l|}{ EULER VECTOR } \\
\hline INPUT & -72 & 64 & 140 & -365 \\
\hline DATABASE & -62 & 74 & 148 & -371 \\
\hline COMPARISON & 1 & 1 & 1 & 1 \\
\hline
\end{tabular}

Table 3

\begin{tabular}{|l|l|l|l|l|}
\hline & \multicolumn{4}{|l|}{ EULER VECTOR } \\
\hline INPUT & -72 & 64 & 140 & -365 \\
\hline DATABASE & 12 & 98 & -28 & 118 \\
\hline COMPARISON & 0 & 0 & 0 & 0 \\
\hline
\end{tabular}

For matching using the comparison matrix, the number of ones and zeros are counted. If all are ones then it is a match and if all are zeros then it is a mismatch. Although, ideally two Euler Codes generated from the same iris should have a comparison matrix of all one's, practically it does not happen because of the noise introduced at various stages. So, we calculate matching score (MSEC) of Euler Code based on the comparison matrix as shown in Equation 11

MSEC $=$ Non-Zero-values $($ Comparison Matrix) $/ 4 \quad$ Eq 11

\subsubsection{Decision Strategy}

Iris recognition systems and algorithms have succeeded in achieving a low false acceptance rate but the rejection rates have remained high. False rejection rate should be as low as possible to make the iris recognition system more practical and adaptable to diverse applications. A template database is prepared. For getting enrolled in the database at least $70 \%$ of iris information should be present, i.e. the noise in the image due to eyelids and eyelashes or the total area covered by the mask should be less than $30 \%$. In the database, templates from three images of an individual are stored. For verifying the identity of an individual, the eye image is detected and if more than $35 \%$ of the noise area is present then the image is not suitable for matching. From the query polar iris image, features are encoded and then the iris templates and Euler Codes are matched using the matching algorithms. A decision strategy is followed as shown below:

1. If all the three MSIT are less than a threshold, thresh-hdmin, then the person is accepted.

2. If all the three MSIT are greater than a threshold, threshhdmax, then the person is rejected.

3. If both the above conditions are not satisfied then:

a. Sort the three matching scores in descending order and

Apply the following equation to get a new matching score MSIT New:

$$
\begin{array}{r}
\operatorname{MSIT}-\mathrm{NEW}=\left(\mathrm{s}_{1} * \operatorname{MSIT}(\max )+\mathrm{s}_{2} * \operatorname{MSIT}(\mathrm{mid})\right. \\
+\mathrm{s}_{3} * \operatorname{MSIT}(\min ) /\left(\mathrm{s}_{1}+\mathrm{s}_{2}+\mathrm{s}_{3}\right)
\end{array}
$$

Where $s_{1}, s_{2}$ and $s_{3}$ are three empirical values such that $s_{1}>s_{2}>$ $\mathrm{s}_{3}$.

This equation gives more weight to the matching score by which a user is accepted and less to the matching score by which a user is rejected

b. Calculate MSEC-Avg, average value of the three matching score of Euler Codes.

c. If MSIT-New and MSEC-Avg are greater than thresholds, thresh-hd-new, and, thresh-ec-avg, then the person is accepted; otherwise the person is rejected.

For our experiments, the values of the matching scores are, threshhd-min is 0.36 , thresh-hd max is $0.40, \mathrm{~s}_{1}, \mathrm{~s}_{2}$, and $\mathrm{s}_{3}$ are $0.3,0.3$ and 0.1 respectively. The value of thresh-hdnew is set to be 0.38 and thresh-ec-avg is 2 .

\section{CONCLUSION}

In this paper a novel iris recognition system is proposed considering both the textural and topological features of an iris image to reduce the false rejection rates. The proposed histogram analysis is applied on a transformed polar iris image to extract textural features and Euler numbers are used to extract the topological features. A decision strategy is proposed to verify the authenticity of an individual.

\section{REFERENCES}

[1] J. Daugman. High confidence visual recognition of persons by a test of statistical independence. IEEE Transactions on Pattern Analysis and Machine Intelligence, 15(11), November 1993.

[2] J. Daugman. Statistical richness of visual phase information: Update on recognizing persons by iris patterns. International Journal of Computer Vision, 45(1):25-38, 2001.

[3] Arijit Bishnu, Bhargab B. Bhattacharya y, Malay K. Kundu, C. A. Murthy "Euler Vector: A Combinatorial Signature for Gray-Tone Images " Proceedings of the International Conference on Information Technology: Coding and Computing (ITCC.02)

[4] "The CASIA iris image database," Available at http://www.sinobiometrics.com.

[5] R BREMANANTH and A CHITRA "New methodology for a person identification system” Sadhana Vol. 31, Part 3, June 2006, pp. 259-276.

[6] J. Daugman. The importance of being random: Statistical principles of iris recognition. Pattern Recognition, 36(2):279- 291, 2003.

[7] Y. Du, C.-I. Chang, H. Ren, F.M. D'Amico, J. Jensen, J., "A New Hyperspectral Discrimination Measure for Spectral Similarity", Optical Engineering, Vol. 43, No. 8, 2004.

[8] C.-I Chang, "An Information Theoretic-based Approach to Spectral Variability, Similarity and Discriminability for Hyperspectral Image Analysis", IEEE Trans. On Information Theory, 46(5), pp. 1927-1932 (2000). 